\title{
EXERCISE RATE ESTIMATION USING A TRIAXIAL ACCELEROMETER
}

\author{
Teddy M. Cheng, Andrey V. Savkin, Branko G. Celler \\ School of Electrical Engineering and Telecommunications, the University of New South Wales, Sydney, NSW 2052, Australia \\ t.cheng@ieee.org,a.savkin@unsw.edu.au,b.celler@unsw.edu.au \\ Steven W. Su \\ Faculty of Engineering, the University of Technology, Sydney, NSW 2007, Australia \\ steven.su@uts.edu.au \\ Ning Wang \\ School of Electrical Engineering and Telecommunications, the University of New South Wales, Sydney, NSW 2052, Australia \\ NingWang@unsw.edu.au
}

\begin{abstract}
Keywords: Wearable sensors, Accelerometer, Exercise intensity, Fundamental frequency estimation, Data fusion, Kalman filtering.

Abstract: In this paper, we propose an algorithm for the estimation of exercise rate during a variety of exercises by using measurements from triaxial accelerometry. The algorithm involves the detection of the periodicity of the body's accelerations, and the detected periods are then fused to form an estimate of exercise rate. Experimental results demonstrate that the algorithm is effective in different modes of exercise. The proposed algorithm will be useful in monitoring training exercises for healthy individuals and rehabilitation exercises for cardiac patients.
\end{abstract}

\section{INTRODUCTION}

Measuring an exerciser's body movements provides a simple and direct way for monitoring and quantifying the intensity of the exercise that is being performed. Accelerometry, in this respect, gives a convenient mean of measuring the body's movements, as well as identifying and classifying movements performed by an exerciser. In particular, portable triaxial accelerometers (TA) are commonly employed (see, e.g., (Chen and Sun, 1997; Kim and Kim, 2008; Karantonis et al., 2006; Tanaka et al., 2007; Asano et al., 2005)). By analyzing the TA measurements, the rate of exercise or activity being performed can be determined. In turn, the exercise rate provides a measure of exercise intensity which is then useful for exercise monitoring.

The objective of this paper is to propose an algorithm for determining the rate of an exercise using a wearable TA. In this study, the exercise rate is termed as the fundamental periodicity or frequency of the locomotion of an exercise, e.g., the stride rate in walk- ing and paddle rate in cycling. The algorithm is universal in the sense that it is capable of estimating the rate regardless of the mode of exercise, and the location of the TA is fixed no matter what exercise is being performed. The algorithm contains two main parts: the first part is to detect the periodicities of the TA measurements, and the second part of the algorithm is to estimate the exercise rate through the use of data fusion techniques. The proposed algorithm can be applied to exercise monitoring in athletics training, monitoring activities of the elderly, and rehabilitation program for the cardiac patients.

\section{METHODS}

\subsection{Equipment}

In this study, the TA for measuring the body accelerations during an exercise is a wireless, single, waistmounted unit, and it measures accelerations in three axes. The sampling rate of the measurements is $50 \mathrm{~Hz}$ 
per channel. The location of the TA is fixed and always mounted on the right-hand-side of the waist of an exerciser. The measurements from the TA are transmitted using a Bluetooth class 1 radio that gives a typical radio range of 100 meters and 30 meters in outdoor and indoor environments respectively. The sampling rate of the measurements is $50 \mathrm{~Hz}$ per channel. To remove any abnormal noise spikes produced by the TA, the raw signals are first median-filtered with a 5-second window. Next, the signals are filtered by a low-pass filter with a cutoff frequency $20 \mathrm{~Hz}$ for removing any high-frequency noises.

\subsection{Exercise Rate Estimation}

To detect the exercise rate, we first estimate the fundamental frequency of each acceleration measurement from the TA. Next, the information of the fundamental frequencies or periodicities of the acceleration measurements are fused together using the Kalman filtering technique.

\subsubsection{Fundamental Frequency Estimation}

The problem of estimating the fundamental frequency of a signal is actively studied in the field of speech processing. Fundamental frequency, or pitch, detection is important for measuring prosodic features in speech for speech recognition and enhancement (Hess, 1983). For these reasons, there are a number of pitch detection algorithms (PDA) reported in the literature (see, e.g. (Ross et al., 1974; Rabiner et al., 1976; Rabiner and Schafer, 1978; de Cheveigne and Kawahara, 2002)). One of the most widely used methods for detecting pitch or fundamental frequency is to use the average magnitude difference function (AMDF) (Ross et al., 1974).

Alternate approaches to estimating the frequency of signals are also proposed in other engineering communities. For instance, in control society, the extended Kalman filtering (EKF) technique is used for estimating the frequency of the fundamental component as well as the amplitudes and phases of the first $m$ harmonic components (Parker and Anderson, 1990). However, such a technique requires a large amount of computational efforts and it is undesirable in real-time applications.

The AMDF of a discrete signal $x_{t}$ is defined by

$$
e_{t}(d)=\frac{1}{N} \sum_{i=1}^{N}\left|x_{t}(i)-x_{t}(i-d)\right|
$$

where $e_{t}(d)$ is the AMDF of lag $d$ calculated at time index $t$ and $N$ is the summation window size in terms of samples. When the lag $d$ is very close or equal to the fundamental period of the signal $x_{t}$, the AMDF (1) will form a dip or a strong local minimum. The AMDF method simply requires one to search for the lowest dip within a given range of lags $D:=\left[d_{\min }, d_{\max }\right]$. Therefore an estimate of the period of the signal $x_{t}$ is given by:

$$
\tau=\hat{d} \times T_{S}
$$

where

$$
\hat{d}:=\min _{d \in D}\left\{e_{t}(d)\right\}
$$

and $T_{S}$ is the sampling period of the discrete signal $x_{t}$. Since the above search may result in the socalled sub-harmonic errors (see e.g., (de Cheveigne and Kawahara, 2002)). These error create undesirable spikes in the period estimates. Therefore, to improve the reliability of the estimates, a causal medianfilter with window length $L$ is employed for removing spikes in the period estimates.

\subsubsection{Data Fusion}

The above procedure allows us to estimate the fundamental periods of 3 acceleration measurements, namely $\tau_{x}(t), \tau_{y}(t)$, and $\tau_{z}(t)$ at time instant $t$. Using the information of these periods and the data fusion technique (Hall and Llinas, 1997; Smyth and Wu, 2007; Tan et al., 2008), an exercise period $T(t)$ (i.e., the inverse of the exercise rate) is to be estimated. Here we have one TA unit mounted on the waist, but it measures accelerations in 3 different directions, giving us 3 measurements for extracting information about the body movement of an exerciser.

In order to perform data fusion for estimating the exercise period $T(\cdot)$, we consider the following discrete-time fusion model

$$
\begin{aligned}
T(k+1) & =T(k)+w(k) \quad \text { for } k=0,1,2, \ldots \\
y(k) & =\left[\begin{array}{l}
y_{1}(k) \\
y_{2}(k) \\
y_{3}(k)
\end{array}\right]=\left[\begin{array}{l}
\tau_{x}(k) \\
\tau_{y}(k) \\
\tau_{z}(k)
\end{array}\right] \\
& =\left[\begin{array}{l}
1 \\
1 \\
1
\end{array}\right] T(k)+\left[\begin{array}{l}
v_{x}(k) \\
v_{y}(k) \\
v_{z}(k)
\end{array}\right] \\
& =: c T(k)+v(k),
\end{aligned}
$$

where $T(\cdot) \in \mathbb{R}$ is the exercise period to be estimated; $y(\cdot) \in \mathbb{R}^{3}$ is the measurement vector consisting of the noisy period measurements $\tau_{x}(\cdot), \tau_{y}(\cdot)$ and $\tau_{z}(\cdot)$ obtained from Section $2.2 .1 ; w(\cdot)$ is a fictitious model noise; and $v_{x}(\cdot), v_{y}(\cdot)$ and $v_{z}(\cdot)$ are the noises on the period measurements. The noise processes $\{w(k)\}$ and $\{v(k)\}$ are assumed to be zero mean white Gaussian noise with covariance $\sigma_{w}(k)$ and covariance matrix $R(k)$ respectively:

$$
E\left[v(k) v(j)^{T}\right]=R(k) \delta(k-j), \text { and } E[v(k) w(j)]=0 .
$$


Our goal is to estimate $T(k)$ using the noisy period measurements $y(k)$ via the Kalman filtering technique (Simon, 2006).

Let $\hat{T}(k)$ be the estimate of $T(k)$ at time instant $k$. The Kalman filter for the fusion model (3) is given by the following equations, which are computed for each time step $k=1,2, \ldots$ :

$$
\begin{aligned}
\hat{T}(k) & =\hat{T}(k-1)+K(k)(y(k)-c \hat{T}(k-1)) \\
K(k) & =p^{-}(k) c^{T}\left(c p^{-}(k) c^{T}+R(k)\right)^{-1} \\
p^{-}(k) & =p^{+}(k-1)+\sigma_{w}(k-1) \\
p^{+}(k) & =(1-K(k) c) p^{-}(k)
\end{aligned}
$$

where $c:=\left[\begin{array}{lll}1 & 1 & 1\end{array}\right]^{T}$. The filter (4) is initialized as follows: $\hat{T}(0)=T_{0}$ and $p^{+}(0)=E\left[\left(T_{0}-\hat{T}(0)\right)^{2}\right]$. The covariance of $\{w(k)\}$ is assumed to be constant $\sigma_{w}(k)=\sigma_{w}$, and the covariance matrix $R(k)$ of the measurement noise $v(k)$ is time-varying and defined as follow:

$$
R_{i, j}(k)= \begin{cases}0, & \text { for } i \neq j \\ r_{i}(k)=\exp \left(\eta\left|y_{i}(k)-\bar{y}(k)\right|\right), & \text { for } i=j\end{cases}
$$

where $\eta>0$ and $\bar{y}(k):=\operatorname{mean}(y(k))$. The $R(k)$ (5) allows us to pay less attention on any measurement $y_{i}(k)$ that is far way from the mean $\bar{y}(k)$.

\section{EXPERIMENTAL RESULTS}

An experimental study was performed to validate the proposed estimation algorithm. Two experimental subjects were requested to exercise on a treadmill and a cycle ergometer. For each kind of exercise, two exercise rates, in terms of period in seconds, were specified. To follow the specified exercise rates, the subjects were instructed to exercise following the periodic audio beeps generated from a digital metronome (Intelli Digital IMT-202 Metronome). The subjects had been given a training session for them to familiarize with the equipments and to get used to exercising at a specific rate given by the digital metronome. We therefore assumed that the subjects could follow the periodic audio beeps from the digital metronome closely throughout the exercise, and the exercise rates were approximately equal to the frequencies of the audio beeps from the digital metronome.

Each subject completed 2 sessions of walking and cycling exercises. In each session, the subject was requested to exercise on an equipment at 2 given rates for 6 minutes continuously: first 3 minutes for one fixed rate and the next 3 minutes for another fixed rate. During the exercise, the body accelerations of
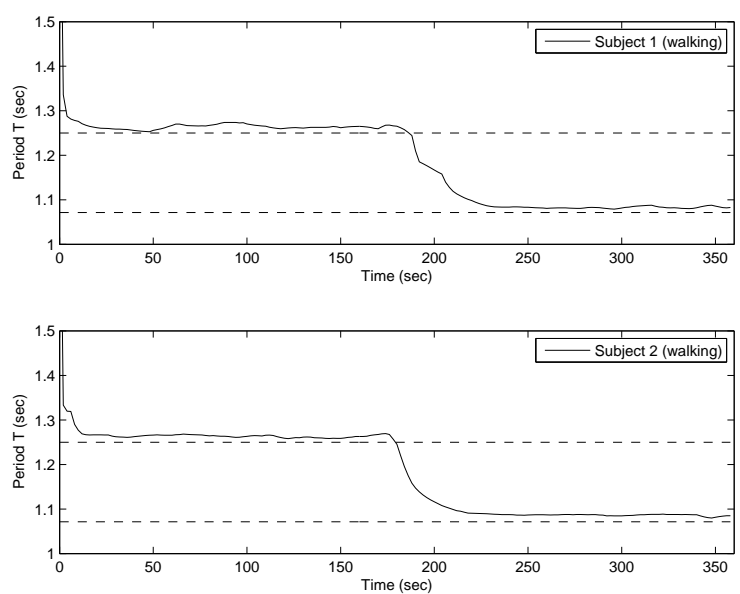

Figure 1: Estimation of walking stride rate using a triaxial accelerometer (at 2 exercise rates: 1.25 and 1.07 secs). (Top) Subject 1, (Bottom) Subject 2.

the subject were recorded by the TA unit as described in Section 2.1.

Table 1 summaries the design parameters for the proposed algorithm in the fundamental frequency detection and data fusion stages.

Table 1: Design parameters for the exercise rate estimation algorithm.

\begin{tabular}{|c|}
\hline AMDF search range in seconds: \\
$D=[0.5,4]$ \\
\hline Causal median filter window length: \\
$L=5$ \\
\hline Kalman filter parameters: \\
$\hat{T}_{0}=2, p^{+}(0)=4$, \\
$\sigma_{w}=0.01, \eta=10$ \\
\hline
\end{tabular}

As shown in Figures 1-2, the proposed algorithm demonstrated to perform well, as the exercise rate estimates from the algorithm closely match with the specified exercise rates for both subjects and for all 2 exercises. The algorithm was able to track the change of exercise rate at the 3-minute mark and settled to the new rate in a reasonable short transient period.

One concern is that the estimated exercise rate of Subject 1 during the first 3 minutes of cycling exercise appears to have some fluctuations (see Figure 2), but this kind of fluctuations were not observed in other exercise rate estimates. This is currently under investigation, it might due to the fact that Subject 1 did not concentrate on following the demands from the digital metronome during the cycling exercise in the first 3 minutes or there is a sensitivity issue of the algorithm that may need to be resolved. 

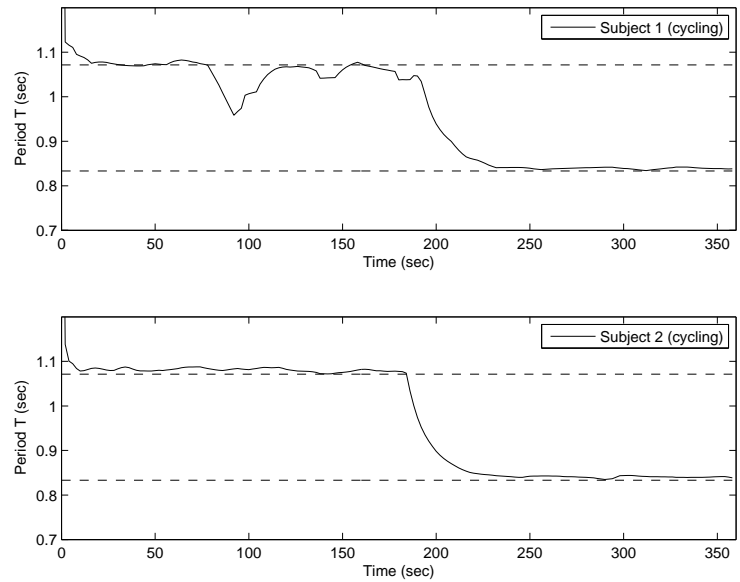

Figure 2: Estimation of cycling pedal rate using a triaxial accelerometer (at 2 exercise rates: 1.07 and $0.83 \mathrm{secs}$ ). (Top) Subject 1, (Bottom) Subject 2.

\section{CONCLUSIONS}

An algorithm for the estimation of exercise rate from triaxial accelerometer measurements was proposed in this paper. The proposed algorithm is universal regardless of the mode of exercise, and it has been experimentally verified in determining the exercise rates of walking and cycling. The algorithm can readily be applied to the monitoring of rehabilitation exercise for the cardiac patients, and training exercise for the athletics. Also, it will be useful in monitoring activities of the elderly and the obese.

\section{ACKNOWLEDGEMENTS}

This work was supported by the Australian Research Council.

\section{REFERENCES}

Asano, M., Tanabe, Y., Watanabe, K., Genno, H., Nemoto, K., Nose, H., and Isawa, M. (2005). Development of an exercise meter using triaxial acceleration data. In Proceedings of Annual International Conference of the IEEE Engineering in Medicine and Biology Society, pages 3731-3734.

Chen, K. Y. and Sun, M. (1997). Improving energy expenditure estimation by using a triaxial accelerometer. Journal of Applied Physiology, 83:2112-2122.

de Cheveigne, A. and Kawahara, H. (2002). YIN, a fundamental frequency estimator for speech and music. Journal of the Acoustical Society of America, 111(4): 1917 - 30.
Hall, D. L. and Llinas, J. (1997). An introduction to multisensor data fusion. Proceedings of the IEEE, 85(1):623.

Hess, W. (1983). Pitch determination of speech signals. Springer-Verlag, Berlin.

Karantonis, D. M., Narayanan, M. R., Mathie, M., Lovell, N. H., and Celler, B. G. (2006). Implementation of a real-time human movement classifier using a triaxial accelerometer for ambulatory monitoring. IEEE Transactions on Information Technology in Biomedicine, 10(1):156-167.

Kim, D. and Kim, H. (2008). Estimation of activity energy expenditure based on activity classification using multi-site triaxial accelerometry. Electronics Letters, 44(4):266- 267.

Parker, P. J. and Anderson, B. D. O. (1990). Frequency tracking of nonsinusoidal periodic signals in noise. Signal Processing, 20(2):127-152.

Rabiner, L., Cheng, M., Rosenberg, A., and McGonegal, C. (1976). A comparative performance study of several pitch detection algorithms. IEEE Transactions on Acoustics, Speech and Signal Processing, ASSP24(5):399-418.

Rabiner, L. and Schafer, R. (1978). Digital processing of speech signals. Prentice-Hall, Englewood Cliffs, NJ.

Ross, M. J., Shaffer, H. L., Cohen, A., Freudberg, R., and Manley, H. J. (1974). Average magnitude difference function pitch extractor. IEEE Transactions on Acoustics, Speech, and Signal Processing, ASSP-22(5):353 -362 .

Simon, D. (2006). Optimal State Estimation: Kalman, $H_{\infty}$, and nonlinear approaches. John Wiley \& Sons, New Jersey.

Smyth, A. and Wu, M. (2007). Multi-rate Kalman filtering for the data fusion of displacement and acceleration response measurements in dynamic system monitoring. Mechanical Systems and Signal Processing, 21(2):706-723.

Tan, H., Wilson, A. M., and Lowe, J. (2008). Measurement of stride parameters using a wearable GPS and inertial measurement unit. Journal of Biomechanics, 41(7):1398-1406.

Tanaka, C., Tanaka, S., Kawahara, J., and Midorikawa, T. (2007). Triaxial accelerometry for assessment of physical activity in young children. Obesity, 15(5):1233-1241. 